\title{
A Time-Domain Active Vibration Control Algorithm for Helicopter Based on a Prior Error Channel Identification Strategy
}

\author{
Yang Lu $(\mathbb{D})$ and Jianbo Feng \\ National Key Laboratory of Rotorcraft Aeromechanics, Nanjing University of Aeronautics and Astronautics, Nanjing 210016, China \\ Correspondence should be addressed to Yang Lu; njluyang@163.com
}

Received 7 August 2017; Revised 13 November 2017; Accepted 11 December 2017; Published 3 January 2018

Academic Editor: Enrico Zappino

Copyright (C) 2018 Yang Lu and Jianbo Feng. This is an open access article distributed under the Creative Commons Attribution License, which permits unrestricted use, distribution, and reproduction in any medium, provided the original work is properly cited.

During the flight tests of Z-11 helicopter, the previous method for active vibration control of helicopter suffered from the problems of slow convergence speed and big residual vibration. In order to mitigate these problems, a new method was proposed on the basis of the previous one. Here, a new error channel modeling method is used, which is named the prior error channel identification strategy. And the active control process and the error channel identification process are simplified to improve performance of the system. Besides, the complexity of the method is reduced. Active vibration control simulations were performed based on a simplified model of helicopter fuselage. The simulation results show that the proposed method has lower residual vibration, faster convergence speed, and better capacity for resisting disturbance compared with the previous method. Finally, an active control of structure response experiment was conducted on a free-free beam, and good inhibitory effect of the vibration response of the freefree beam was obtained. The results illustrate that the proposed method based on the prior error channel identification strategy is effective.

\section{Introduction}

Excessive vibration on a helicopter airframe can cause physical and psychological discomfort to passengers and crew, fatigue and damage to the aircraft structure and on-board equipment, and decreased operational capabilities of the aircraft $[1,2]$. Traditional passive vibration control systems are often relatively large in volume, are heavy in weight, and cannot adapt to the changes of vibration frequency [3-5]. And most of all, the vibration control effect is not satisfactory. Active vibration control approaches for helicopter such as higher harmonic control (HHC), individual blade control (IBC), actively controlled flaps (ACF), active twist rotor (ATR), and active control of structure response (ACSR) can suppress unwanted vibration adaptively and make up for the weaknesses of passive techniques [6-9]. Among these approaches, ACSR has received considerable attention for its simple system components and stable performance and it has been used in helicopters such as EH-101, UH-60, and EC225/EC725 [10-12].
ACSR is based on the principle of superposition; by introducing a secondary vibration source in the original control system, the primary undesired vibration could be suppressed when the output of the secondary vibration source is adjusted through certain control law. Two kinds of adaptive control algorithms are commonly used: frequency-domain algorithm and time-domain algorithm. Due to its simplicity and good stability, frequency-domain algorithm has been successfully applied in ACSR systems of helicopters like UH-60 and S-92 [13, 14]. Even so, because of its own characteristic, the update speed of frequency-domain algorithm is slow, and its adaptability is insufficient when helicopters are in maneuvering flight, while time-domain algorithm has the advantage of quick update speed and does not need to change the signal form time-domain to frequency-domain. In recent years, some researchers have applied time-domain algorithm in helicopters like EC135, EC145, and SURION [15-17].

In addition, authors have applied a time-domain algorithm in the active vibration control flight tests of Z-11 in 2011 [18]. The algorithm used in the flight tests is developed by 
Yang et al. [19], which is an online secondary path modeling method using an auxiliary random noise. Due to the complex external environment during the flight tests, the convergence speed of the algorithm was slow, and the control performance was unsatisfactory because of the big residual vibration.

The basic online error channel identification method using an auxiliary random noise was first proposed by Eriksson and Allie [20] in 1989. However, due to the interaction between the error channel filter and the active control filter, the overall performance of the system is degraded. In order to improve the performance of Eriksson's method, Zhang et al. introduced a third adaptive filter to eliminate the bad effect of the error channel identification process on the active control process [21]. Based on of Zhang's method, Yang et al. introduced a fixed filter to eliminate the influence of the active control process on the error channel identification process [19].

To mitigate the problems during the flight tests and improve the performance of the control system, in this paper a new method is proposed based on Yang's method. The organization of this paper is as follows: Section 2 gives a brief overview of the existing methods. In Section 3, a new method is proposed based on the prior error channel identification strategy. Section 4 presents three simulations to demonstrate the performance of the proposed method. In Section 5, an experiment of ACSR on a free to free beam based on DSP is provided. Section 6 gives the conclusions of this paper.

\section{Briefing of Existing Methods}

In order to better describe the proposed method, in this section, two important existing online error channel identification methods are reviewed briefly. These methods are based on FxLMS algorithm, and some useful concepts are introduced.

2.1. Eriksson's Method. The block diagram of Eriksson's method is shown in Figure 1. As can be seen, a random noise $v(n)$, which is independent of the reference signal $x(n)$, is superposed with the output of the controller, and $e(n)$ is the residual vibration. The vector $\mathbf{P}(n)$ denotes the impulse response vector of the primary channel. The vector $\mathbf{S}(n)$ denotes the impulse response vector of the error channel. $\widehat{\mathbf{S}}(n)$ is an identification FIR filter of length $M$, and $\mathbf{W}(n)$ is an identification FIR filter of length $L$. The signal $d(n)$ is the output of $\mathbf{P}(n)$, and $y(n)$ is the output of $\mathbf{W}(n)$.

The error signal for $\mathbf{W}(n)$ is expressed as

$$
e(n)=\left[d(n)-y^{\prime}(n)\right]+u(n),
$$

where $y^{\prime}(n)=\mathbf{S}(n)^{T} \mathbf{Y}(n), u(n)=\mathbf{S}(n)^{T} \mathbf{V}(n), \mathbf{Y}(n)=$ $[y(n) \cdots y(n-M+1)], y(n)=\mathbf{W}(n-1)^{T} \mathbf{X}(n)$.

The coefficients of the filter $\mathbf{W}(n)$ are updated by FxLMS algorithm.

$$
\mathbf{W}(n+1)=\mathbf{W}(n)+\mu_{w} \mathbf{X}^{\prime}(n) e(n),
$$

where $\mu_{w}$ is the step size of the active control process, $\mathbf{X}^{\prime}(n)=$ $\left[x^{\prime}(n) \cdots x^{\prime}(n-M+1)\right]^{T}, x^{\prime}(n)=\widehat{\mathbf{S}}(n)^{T} \mathbf{X}(n)$.

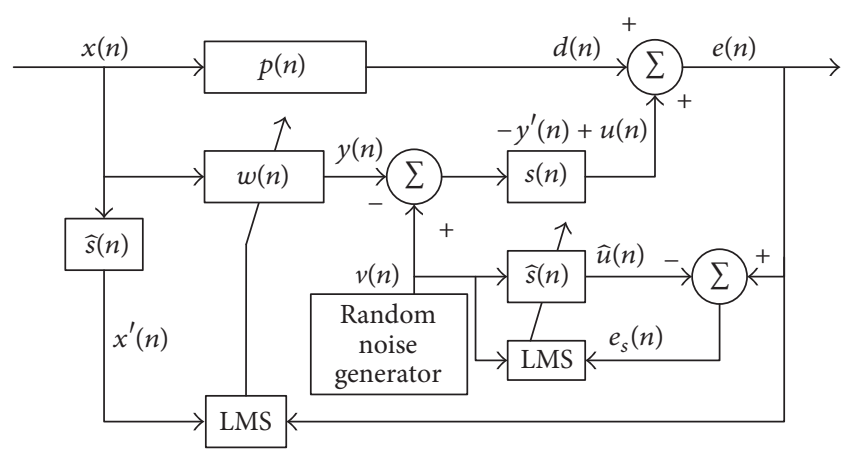

FIGURE 1: Eriksson's method with online error channel identification.

The error signal for $\widehat{\mathbf{S}}(n)$ is expressed as

$$
e_{s}(n)=\left[d(n)-y^{\prime}(n)\right]+[u(n)-\widehat{u}(n)],
$$

where $\widehat{u}(n)=\widehat{\mathbf{S}}(n)^{T} \mathbf{V}(n)$.

The coefficients of the filter $\widehat{\mathbf{S}}(n)$ are updated by LMS algorithm.

$$
\widehat{\mathbf{S}}(n+1)=\widehat{\mathbf{S}}(n)+\mu_{s} \mathbf{V}(n) e_{s}(n),
$$

where $\mu_{s}$ is the step size of the error channel process.

2.2. Yang's Method. Figure 2 shows the block diagram of Yang's method. In Yang's method, the coefficients of the filter $\mathbf{W}(n)$ are updated by FxLMS algorithm as well.

$$
\mathbf{W}(n+1)=\mathbf{W}(n)+\mu_{w} \mathbf{X}^{\prime}(n) e_{w}(n),
$$

where $e_{w}$ is the error signal for the active control filter $\mathbf{W}(n)$, and $e_{w}(n)=\left[d(n)-y^{\prime}(n)\right]+[u(n)-\widehat{u}(n)]$.

The error signal for $\widehat{\mathbf{S}}(n)$ and $\mathbf{H}(n)$ is expressed as

$$
e_{s h}(n)=e_{w}-\widehat{d}(n)+\widehat{y}^{\prime}(n),
$$

where $\widehat{y}^{\prime}(n)=\widehat{\mathbf{S}}(n)^{T} \mathbf{Y}(n), \widehat{d}(n)=\mathbf{H}(n)^{T} \mathbf{X}(n)$.

The coefficients of the filter $\widehat{\mathbf{S}}(n)$ are updated by LMS algorithm.

$$
\widehat{\mathbf{S}}(n+1)=\widehat{\mathbf{S}}(n)+\mu_{s} \mathbf{V}(n) e_{s h}(n),
$$

where $\mu_{s}$ is the step size of the error channel process.

The coefficients of the filter $\mathbf{H}(n)$ are updated by LMS algorithm.

$$
\mathbf{H}(n+1)=\mathbf{H}(n)+\mu_{h} \mathbf{X}(n) e_{s h}(n),
$$

where $\mu_{h}$ is the step size of the parallel identification process.

\section{Proposed Method}

From Yang's method, in ideal condition the interaction between the active control process and the error channel identification process can be eliminated. However, the error 


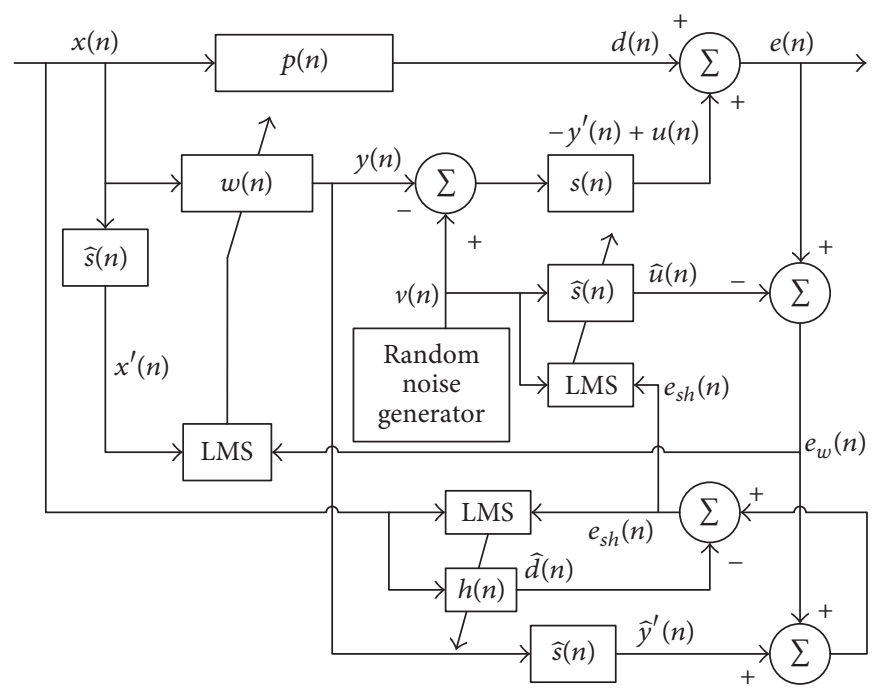

FIGURE 2: Yang's method with online error channel identification.

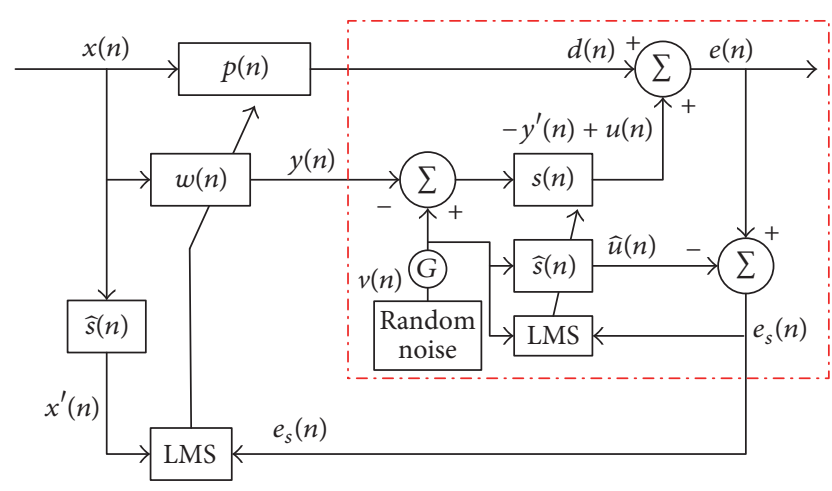

FIGURE 3: Proposed algorithm with prior error channel identification.

channel identification process and the parallel identification process may interact with each other again. In fact, the improvement of the control performance is limited. Moreover, the problem of the auxiliary random noise appearing in the residual vibration is not addressed.

To overcome the above disadvantages, a new method based on a prior error channel identification strategy is constructed. A block diagram of the proposed algorithm is shown in Figure 3.

Firstly, a prior error channel identification strategy is designed to obtain an accurate error channel model while alleviating increment of the residual vibration due to the auxiliary random noise. Secondly, the active control process and the error channel identification process are simplified, and the modified error signal for the active control filter and the error channel filter is introduced to improve the performance of the system.

3.1. Prior Error Channel Identification Strategy. As can be seen from Figure 3, the error signal is $e(n)=\left[d(n)-y^{\prime}(n)\right]+u(n)$, and it comprises two components; that is, $e(n)=e_{1}(n)+e_{2}(n)$, where $e_{1}(n)$ is the component due to $x(n), e_{1}(n)=[\mathbf{P}(n)-$ $\mathbf{W}(n) \mathbf{S}(n)] \mathbf{X}(n)$, and $e_{2}(n)$ is the component due to $v(n)$, $e_{2}(n)=\mathbf{S}(n)^{T} \mathbf{V}_{r}(n)$. From the point of view of system identification, $e_{1}(n)$ is the interference to the updating of $\widehat{\mathbf{S}}(n)$, while $e_{2}(n)$ is the interference to the updating of $\mathbf{W}(n)$. In ideal conditions, when $\mathbf{W}(n)=\mathbf{P}(n) / \mathbf{S}(n), e_{1}(n)=0$, and $e(n)=e_{2}(n)=\mathbf{S}(n)^{T} \mathbf{V}_{r}(n)$, the error signal is fully relative to $v(n)$, and it cannot be controlled. A large auxiliary random noise will greatly increase the residual vibration and be harmful to the convergence of $\mathbf{W}(n)$, while a small auxiliary random noise will not be beneficial to the error channel identification. This problem will always exist, if the active control filter and the error channel identification filter are updated simultaneously. In order to obtain an accurate error channel model and alleviate the increment of the residual vibration due to the auxiliary random noise, a prior error channel identification strategy is proposed.

From Figure 3, $v_{r}(n)=v(n) * G$, where $G$ is amplification factor of the auxiliary random noise. In this paper, assume $v(n)$ is big enough for the error channel identification. During the period of the prior identification of the error channel (the red dash dot box of Figure 3), $G=1$, after that $0<G<1$. The value of $G$ should be chosen according to the error channel characteristics and the performance of the active control system in simulations or experiments. If the error channel is time invariant, the value of $G$ can be zero, and in this situation the whole system will degrade into an offline error channel identification system. If the error channel is time variant, the value of $G$ should be chosen carefully according to the performance of the active control system. At the beginning of the running algorithm, the error channel identification process runs alone; a bigger auxiliary random noise $(G=$ 1 ) is used to identify the error channel quickly. After the accomplishment of the error channel identification, the active control process and the error channel identification process run at the same time. Due to the good initial value of the error 
channel identification process, the identification work can be done using a smaller auxiliary random noise.

Above all, this strategy can alleviate the increment of the residual vibration due to the auxiliary random noise, on the basis of the fast and effective error channel identification.

Besides, using the prior error channel identification strategy can separate the error channel identification process and the active control process, thus avoiding the interaction between them.

3.2. Process of the Active Control. After filtering through $\widehat{\mathbf{S}}(n)$, $x^{\prime}(n)$ becomes the new reference signal. As shown in Figure 3, the weights of the active control filter $\mathbf{W}(n)$ are updated as follows:

$$
\begin{aligned}
\mathbf{W}(n+1) & =\mathbf{W}(n)+\mu_{w} \mathbf{X}^{\prime}(n) e_{s}(n) \\
e_{s}(n) & =\left[d(n)-y^{\prime}(n)\right]+[u(n)-\widehat{u}(n)],
\end{aligned}
$$

where $y^{\prime}(n)=\mathbf{S}(n)^{T} \mathbf{Y}(n), \widehat{u}(n)=\mathbf{S}(n)^{T} \mathbf{V}_{r}(n), \mathbf{Y}(n)=$ $[y(n) \cdots y(n-M+1)], y(n)=\mathbf{W}(n-1)^{T} \mathbf{X}(n)$. And in ideal conditions, $\widehat{\mathbf{S}}(n)=\mathbf{S}(n), u(n)=\widehat{u}(n), e_{s}(n)=d(n)-y^{\prime}(n)$, $e_{s}(n)$ is fully relative with $x(n)$; then impact of the auxiliary noise $v(n)$ on the active control process is eliminated.

To sum up, by using the proposed algorithm, these goals can be achieved: (1) the complexity of the method is reduced by avoiding the introduction of the third active control filter mentioned in Yang's method; (2) the influence of the auxiliary random noise on the active control process is eliminated.

3.3. Process of the Error Channel Identification. According to Yang's method, after introducing the filter of the parallel identification process and the filter of the error channel, the interaction between the active control process and the error channel identification process is eliminated. Nonetheless, using $e_{s h}(n)$ as the error signal to update the weights of the parallel process filter, it will cause the interaction between the parallel channel identification process and the error channel identification process. That is to say, the interaction with the error channel identification process has not been eliminated, it just transfers from the active control process to the parallel channel identification process.

Detailed analysis is as follows. From Yang's method, the update of the weights of the parallel identification filter $\mathbf{H}(n)$ is given by (8). From (6), it can be seen that $e_{s h}(n)$ cannot be fully relative to the reference signal. It still has connection with the auxiliary random noise $v(n)$ and the weights of the error channel filter $\widehat{\mathbf{S}}(n)$, which illustrates that the influence of the error channel identification process on the parallel channel identification process has not been eliminated.

To solve the problem, the proposed algorithm uses $e_{s}(n)$ as the error signal to update the weights of the error channel identification filter on the basis of nonuse of the parallel identification filter. $e_{s}(n)$ is expressed as

$$
\begin{aligned}
e_{s}(n) & =\left[d(n)-y^{\prime}(n)\right]+[u(n)-\widehat{u}(n)] \\
\widehat{\mathbf{S}}(n+1) & =\widehat{\mathbf{S}}(n)+\mu_{s} \mathbf{V}(n) e_{s}(n) .
\end{aligned}
$$

Note that $d(n)-y^{\prime}(n)$ is the influence of the active control process on the error channel identification process. In ideal conditions, when $\mathbf{W}(n)$ converges to $\mathbf{P}(n) / \mathbf{S}(n), d(n)=y^{\prime}(n)$, $e_{s}(n)$ becomes fully relative to $v(n)$. Thus, the influence of the active control process on the error channel identification process is eliminated.

3.4. Summary of the Proposed Method and Computational Complexity Analysis. According to Figure 3, the proposed method can be summarized as follows:

$$
\begin{aligned}
v_{r}(n) & =v(n) * G \\
d(n) & =\mathbf{P}(n)^{T} \mathbf{X}(n) \\
y(n) & =\mathbf{W}(n)^{T} \mathbf{X}(n) \\
y^{\prime}(n) & =\mathbf{S}(n)^{T} \mathbf{Y}(n) \\
u(n) & =\mathbf{S}(n)^{T} * \mathbf{V}_{r}(n) \\
\widehat{u}(n) & =\widehat{\mathbf{S}}(n)^{T} * \mathbf{V}_{r}(n) \\
x^{\prime}(n) & =\widehat{\mathbf{S}}(n)^{T} \mathbf{X}(n) \\
e(n) & =d(n)-y^{\prime}(n)+u(n) \\
e_{s}(n) & =e(n)-\widehat{u}(n) \\
\mathbf{W}(n+1) & =\mathbf{W}(n)+\mu_{w} \mathbf{X}^{\prime}(n) e_{s}(n) \\
\widehat{\mathbf{S}}(n+1) & \widehat{\mathbf{S}}(n)+\mu_{S} \mathbf{V}_{r}(n) e_{s}(n)
\end{aligned}
$$

Table 1 gives the comparison of the proposed method with the existing methods in computational complexity. Here, $N$ stands for the length of the parallel process filter, in the following simulations, $N=L=M$. As it can be seen, the computational complexity of the proposed method is less than Yang's method; this is because the proposed method does not use the third adaptive filter. The computational complexity of the proposed method is a little more than Eriksson's method; this is because of the introduction of $G$, and it is used to eliminate the interaction between the active control process and the error channel identification process.

\section{Simulation Results and Analysis}

In order to study thecharacteristics of the proposed method and check out its effectiveness in helicopter ACSR, three kinds of simulations are performed based on MATLAB in this section. For intuitively measuring the accuracy of the error channel identification, the relative error of the error channel identification can be defined as

$$
\Delta S=10 \log _{10}\left\{\frac{\sum_{i=0}^{M-1}\left[\mathbf{S}_{i}(n)-\mathbf{S}_{i}^{\prime}(n)\right]^{2}}{\sum_{i=0}^{M-1}\left[\mathbf{S}_{i}(n)\right]^{2}}\right\} .
$$

In this paper, if $\Delta S \leq-40 \mathrm{~dB}$, we consider the error channel identification is accomplished. 
TABLE 1: Computational complexity.

\begin{tabular}{lccr}
\hline & Multiplications & Additions & Total \\
\hline Eriksson algorithm & $2 L+3 M+2$ & $2 L+3 M-1$ & $4 L+6 M+1$ \\
Yang's algorithm & $2 L+4 M+2 N+14$ & $2 L+4 M+2 N+16$ & $4 L+8 M+4 N+30$ \\
Proposed algorithm & $2 L+3 M+3$ & $2 L+3 M-1$ & $4 L+6 M+2$ \\
\hline
\end{tabular}

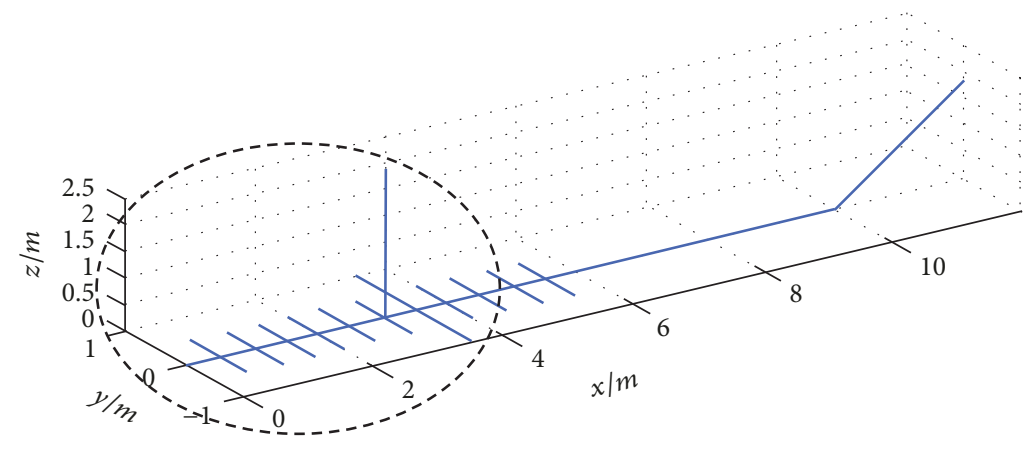

(a) The whole fuselage

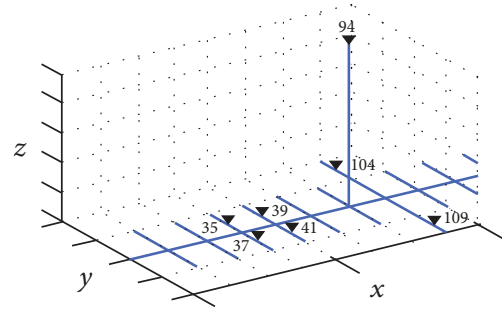

(b) Front part of the fuselage

FIGURE 4: Simplified FEM model of helicopter.

4.1. Simulation Model. As shown in Figure 4, a finite element model of free-free combination beam is built using ANSYS, and it is a simplified model of helicopter fuselage. The parameters of the model beam are as follows: $m=6.114 \times$ $10^{3} \mathrm{~kg}, \rho=7.8 \times 10^{3} \mathrm{~kg} / \mathrm{m}^{3}, E=210 \mathrm{Gpa}, \mu=0.3$, where $m$ stands for mass, $\rho$ stands for density, $E$ stands for elasticity modulus, and $\mu$ stands for Poisson's ratio. Figure 4 (b) shows the amplified front part of the model. As shown in the figure, the extraneous force is loaded at the node 94 to simulate the exciting force on the rotor hub; the control force is loaded at the node 109; the acceleration response of the node 37 is used as the error signal, to evaluate the effectiveness of the active control system in vibration reduction. Take the former seven order modes of the system as the system response.

4.2. Case 1: Single Frequency Input. The main frequency of helicopter's vibration is blade passage frequency which is the product of rotor rotating speed and number of blades. In this case, the reference signal is sine signal with frequency of $17 \mathrm{~Hz}$ and amplitude of $3000 \mathrm{~N}$. A zero-mean white Gaussian noise of variance 1 is used for the error channel identification. Sampling frequency is $1000 \mathrm{~Hz}$; the order of the adaptive filters is 32 . The parameters of the proposed method are $\mu_{w}=$ $0.002, \mu_{s}=0.001$,

$$
\begin{aligned}
& G=1 \quad n=0-2000 \\
& G=0.1 \quad n=2000-40000 .
\end{aligned}
$$

The parameters of Eriksson's method are the same as the proposed method, and the parameters of Yang's method are the same as the proposed method, except for $\mu_{h}=0.003$. These parameters are chosen during the simulation according to the performance of vibration reduction.

Comparisons are made in convergence speed and performance of active vibration control.

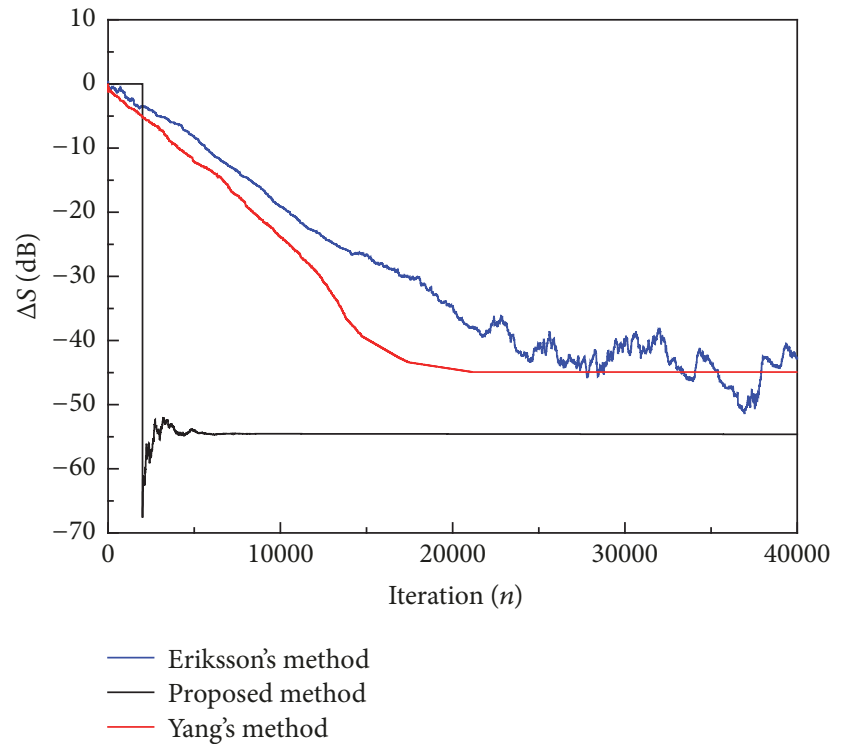

FIgURE 5: Performance comparison of error channel identification.

Figure 5 presents the identification errors of the three methods. It can be seen that the identification error of the proposed method drops to $-55 \mathrm{~dB}$ after 7000 iterations and comes to a steady state, and Yang's method needs about 20000 iterations to reach a steady state and the identification error is $-45 \mathrm{~dB}$. While Eriksson's method needs much more time, and it is not stable. The result indicates that the proposed method can lead to a quick acquisition of accurate error channel identification.

Figure 6 presents the acceleration of the controlled point of Eriksson's and Yang's method, and Figure 7 presents the acceleration of the controlled point of the proposed method. It is hard to distinguish if Figures 6 and 7 are put in one figure. 


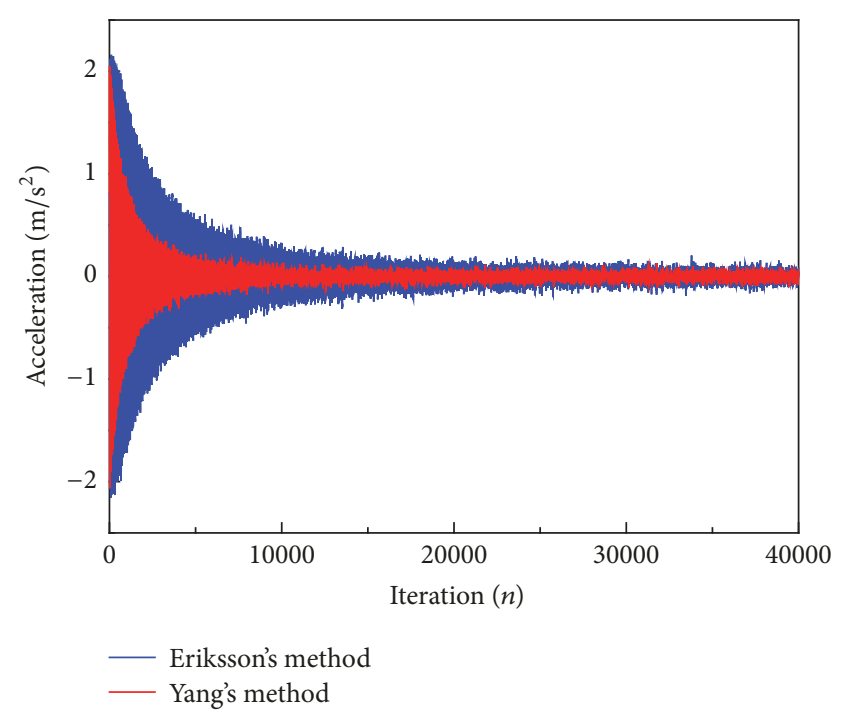

FIGURE 6: Acceleration of the controlled point of existing methods.

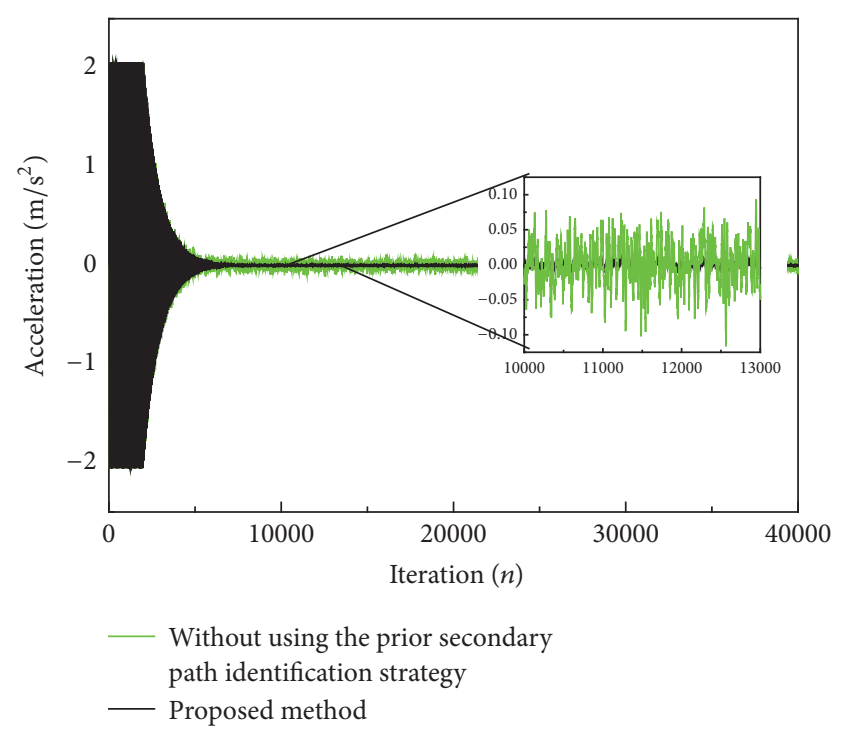

FIGURE 7: Acceleration of the controlled point of the proposed method.

It can be seen from Figures 6 and 7, Eriksson's method needs about 18000 iterations to come to a steady state, and its residual vibration is about $0.12 \mathrm{~m} / \mathrm{s}^{2}$, while Yang's method needs about 8000 iterations to come to a steady state, and its residual vibration is about $0.1 \mathrm{~m} / \mathrm{s}^{2}$. However, the proposed algorithm just needs 7000 iterations to reach the steady state. Besides, the residual vibration is only about $0.01 \mathrm{~m} / \mathrm{s}^{2}$. From the comparison, a conclusion can be made: the proposed method has better performance than both Eriksson's and Yang's method no matter in convergence speed or in steady residual vibration.

From further analysis of Figure 7 , it can be seen that the residual vibration is $0.1 \mathrm{~m} / \mathrm{s}^{2}$ without using the prior error channel identification strategy; after using the prior error channel identification strategy, the residual vibration is

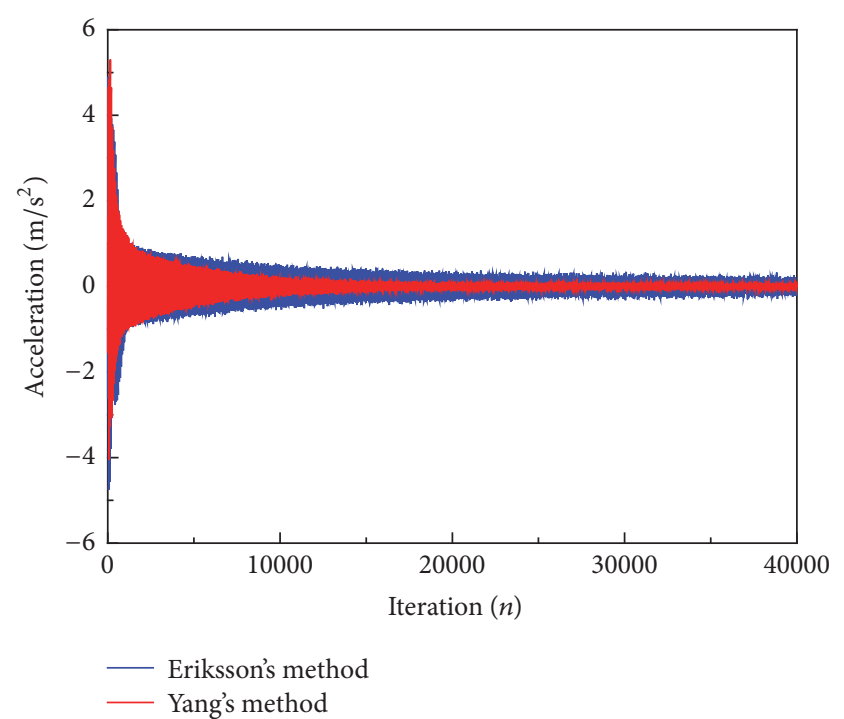

FIGURE 8: Acceleration of the controlled point of existing methods.

only $0.01 \mathrm{~m} / \mathrm{s}^{2}$, which indicates that the prior error channel identification strategy can efficiently reduce the influence of auxiliary random noise on the residual vibration.

4.3. Case 2: Multifrequency Input. Blade passage frequency is not the only main frequency part of helicopter' vibration. In order to simulate the vibration more precisely, the reference signal is set as follows:

$$
f=3000 \sin (2 \pi \times 17 \times t)+1500 \sin (2 \pi \times 34 \times t) .
$$

The order of the adaptive filters is 32 . The step size of the active control filter is $\mu_{w}=0.001$ in these methods, and the other parameters of the methods are the same as case 1.

Figure 8 presents the residual vibration of Eriksson's and Yang's method under multifrequency input. Figure 9 presents the residual vibration of the proposed method.

From the comparison of Figure 8 and Figure 9, it can be seen that the proposed method achieves higher vibration reduction as compared with the other two methods.

As can be seen from Figure 9(a), the part from 0 to 2000 iterations is in the uncontrolled state, with the acceleration of about $2.25 \mathrm{~m} / \mathrm{s}^{2}$. After being controlled, the acceleration of the controlled point reduces quickly, which illustrates the effectiveness of the proposed method under multifrequency input.

From the comparison of Figure 9(a) and Figure 9(b), it is obvious that the controlled point's acceleration using the prior error channel identification strategy is much smaller. Figure 10 shows the vibration spectral analysis of the controlled point at the steady state, which intuitively demonstrate the effectiveness of the prior error channel identification for reducing the influence of auxiliary random noise on the residual vibration.

From Figure 10, it can be seen that the proposed method can reduce the residual vibration to about $0.01 \mathrm{~m} / \mathrm{s}^{2}$ at $17 \mathrm{~Hz}$, and $0.07 \mathrm{~m} / \mathrm{s}^{2}$ at $34 \mathrm{~Hz}$. From comparison of Figure 10 (a) and 


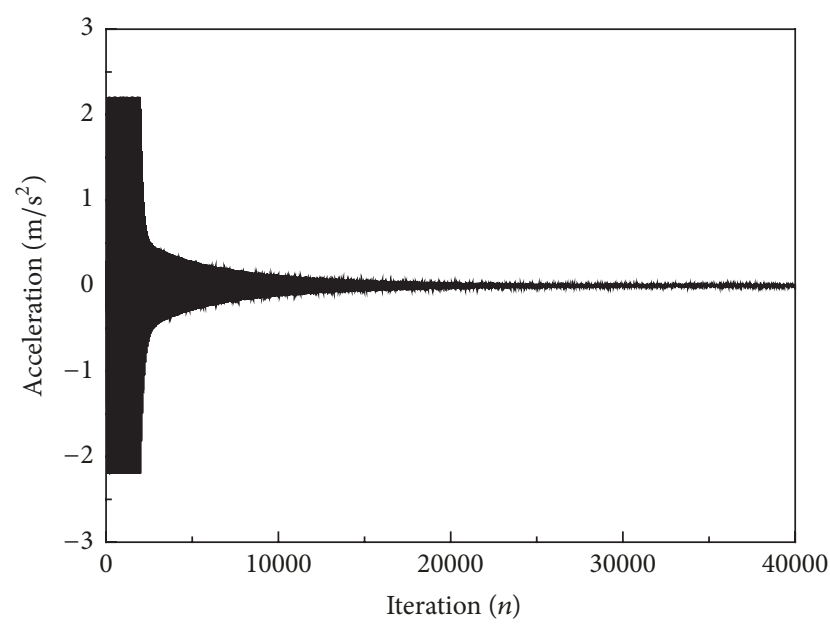

(a) Using the prior error channel identification strategy

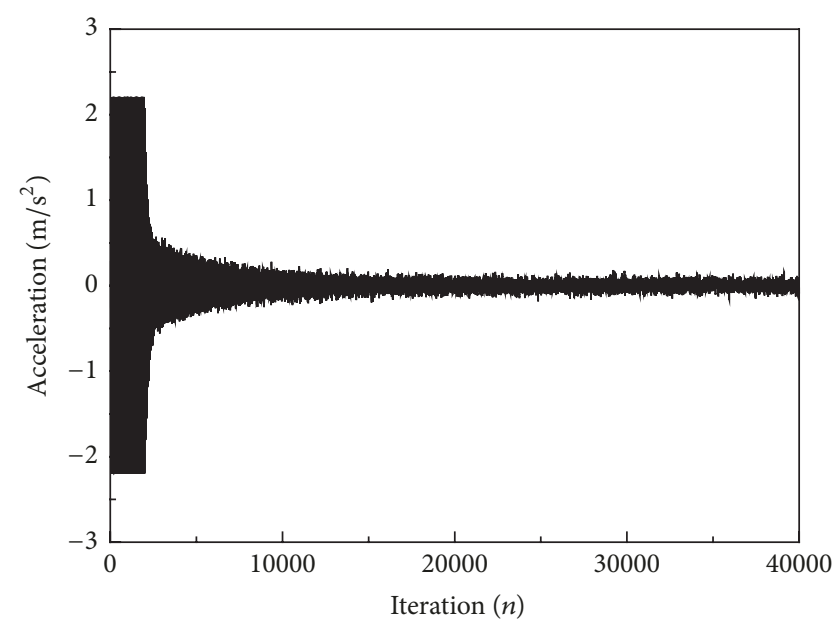

(b) Without using the prior error channel identification strategy

FIgURE 9: Acceleration of the controlled point of proposed method.

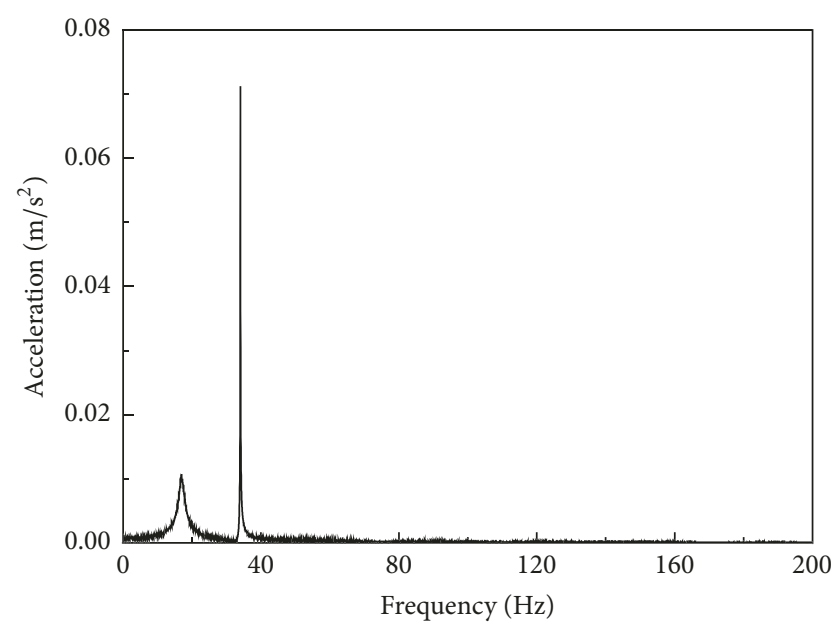

(a) Using the prior error channel identification strategy

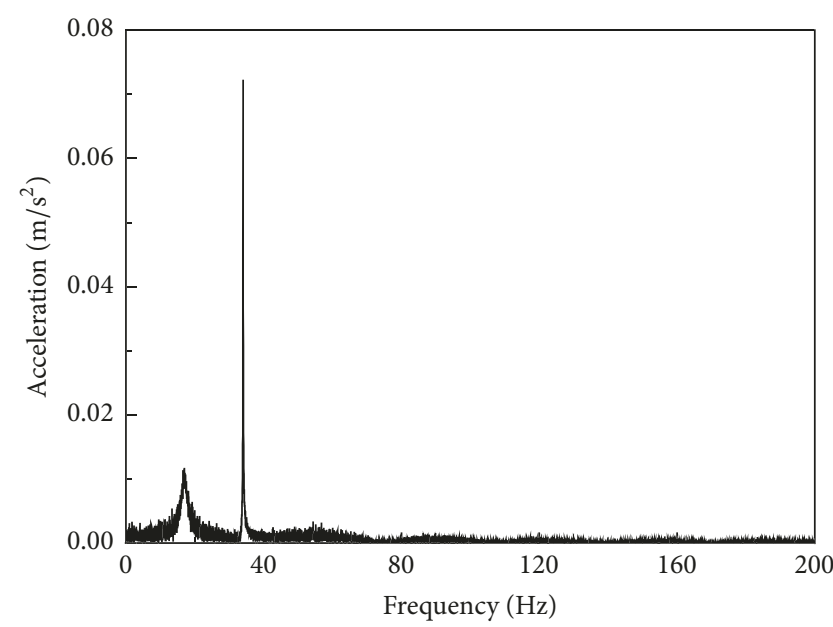

(b) Without using the prior error channel identification strategy

FIGURE 10: Frequency spectrum of controlled point of proposed algorithm.

Figure 10(b), it can be seen that the random component of the residual vibration is reduced dramatically after using the prior error channel identification strategy.

4.4. Case 3: Single Frequency Input with Error Channel Variation. During helicopter maneuvering flight, the transfer function of error channel is varying because of complex external disturbances. In order to simulate this case, the variation of error channel is the same as that of [19], and the reference signal, the auxiliary noise, and the error channel before variation are the same as case 1 . The simulation results are shown as Figures 11 and 12.

As can be seen from Figure 11, the proposed method can reduce the identification error to about $-55 \mathrm{~dB}$ in 3000 iterations after error channel variation. However, Yang's method needs about 14000 iterations to reduce to $-44 \mathrm{~dB}$. It is apparent that the error channel identification of the proposed method has stronger capability in resisting disturbance.

From Figure 12, it can be seen that Yang's method needs 16000 iterations to come to a steady state after error channel variation. However, the proposed method only needs 3000 iterations to reach the steady state, and at the same time the residual vibration is not enlarged. Comparison of Figure 12(a) and Figure 12(b) illustrates the superiority of the proposed method in disturbance resistance.

\section{Active Control Experiment and Results}

To verify the effectiveness of the proposed method, an experiment of active control of structure response on a free-free beam is performed. Based on the embedded digital signal processor (DSP), using the proposed method, an active control system with single input and single output is built for experimental study. 


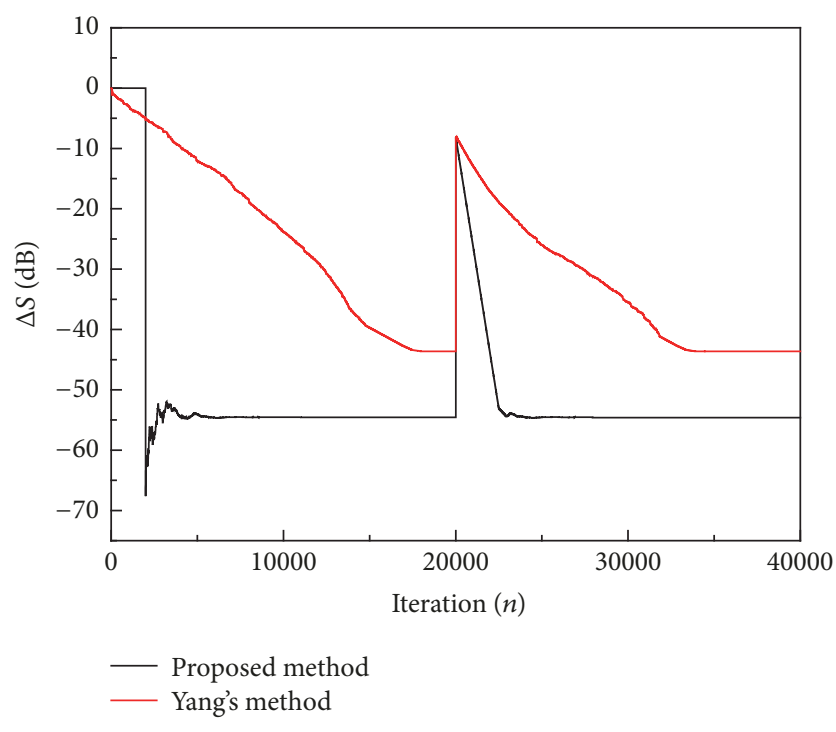

FIGURE 11: Performance comparison of error channel identification.

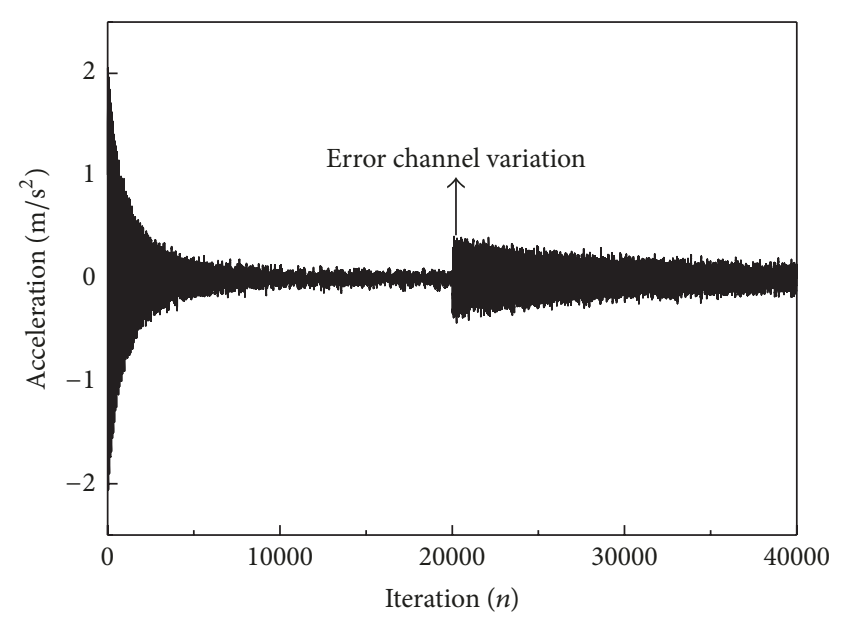

(a) Yang's method

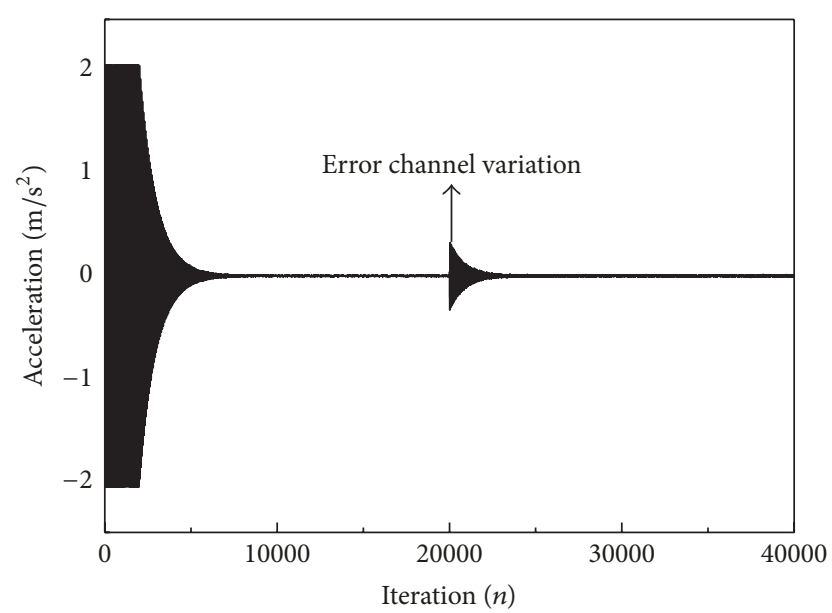

(b) Proposed method

FIgURE 12: Performance comparison between Yang's method and the proposed method with error channel variation.
5.1. Experimental Model and Set-Up. The length of the freefree beam is $3000 \mathrm{~mm}$, and its width and height are $60 \mathrm{~mm}$ and $20 \mathrm{~mm}$, respectively. It is made of steel with the mass of $26.8 \mathrm{~kg}$. Besides, the former three orders of the bending modal frequencies are $11.9 \mathrm{~Hz}, 35 \mathrm{~Hz}$, and $61 \mathrm{~Hz}$. Figure 13 shows the schematic diagram of the experimental system. The free-free beam is used to simulate the helicopter airframe, and the vibration generated by the exciter is used to simulate the rotor hub disturbance force. At first, two sine signals are output by the signal generator. One is amplified by the power amplifier to drive the exciter and the other acts as the reference signal through analog-to-digital conversion. The error signal comes from the acceleration sensor on the beam and is sent to the data acquisition card through CM3508. The error signal and the reference signal as input signals are all sent to the DSP controller and to the upper computer as well. The control signal which comes from DSP is sent to the power amplifier firstly, and then it is used to drive the actuator.

The experimental set-up includes the following: (a) one signal generator; (b) two power amplifiers, type: HEA-500G; (c) one oscilloscope, type: DF4328; (d) one exciter, type: HEV-500; (e) one dynamic signal conditioner, type: CM3508; (f) one ICP acceleration sensor, type: LC0119; (g) one NI data acquisition card; (h) one embedded digital signal processor, type: TMS320C6747; (i) one upper computer. Figure 14 shows the diagram of the experimental set-up.

5.2. Program Implementation. The proposed algorithm is programmed under Code Composer Studio (CCS) v5.2. After compiling, the executable file(.out) is download to DSP C6747 for execution. The flow chart of the program running in DSP is as in Figure 15.

As shown in Figure 15, after system initialization, the control parameters such as $\mu_{w}$ and $\mu_{s}$ are initialized. Then the error channel identification process runs alone with a bigger auxiliary random noise $(G=1)$. After the accomplishment of the error channel identification, the active control process and the error channel identification process run at the same time with a smaller auxiliary random noise $(0<G<1)$ and a good initial value of the error channel filter. At last the active control system converges.

5.3. Experimental Results and Analysis. The reference signal is a sine signal with amplitude $2 \mathrm{~V}$ and frequency $12 \mathrm{~Hz}$. The order of the adaptive filters is 64 , and the initial values are set to zero. The sampling frequency is $1000 \mathrm{~Hz}$. The parameters of the proposed method are $\mu_{w}=0.005, \mu_{s}=0.004$,

$$
\begin{aligned}
G & =1 \quad T=4-7 \mathrm{~s} \\
G & =0.1 \quad T=7-40 \mathrm{~s} .
\end{aligned}
$$

The parameters of Yang's method are the same as the proposed method except for $\mu_{h}=0.002$. These parameters are chosen during the experiment according to the performance of the system.

The time history of Yang's method is shown in Figure 16(a). The first $4 \mathrm{~s}$ corresponds to the uncontrolled state, and the active controller starts working from $T=4 \mathrm{~s}$. It can be seen that after about $10 \mathrm{~s}$ of the adjustment process, 


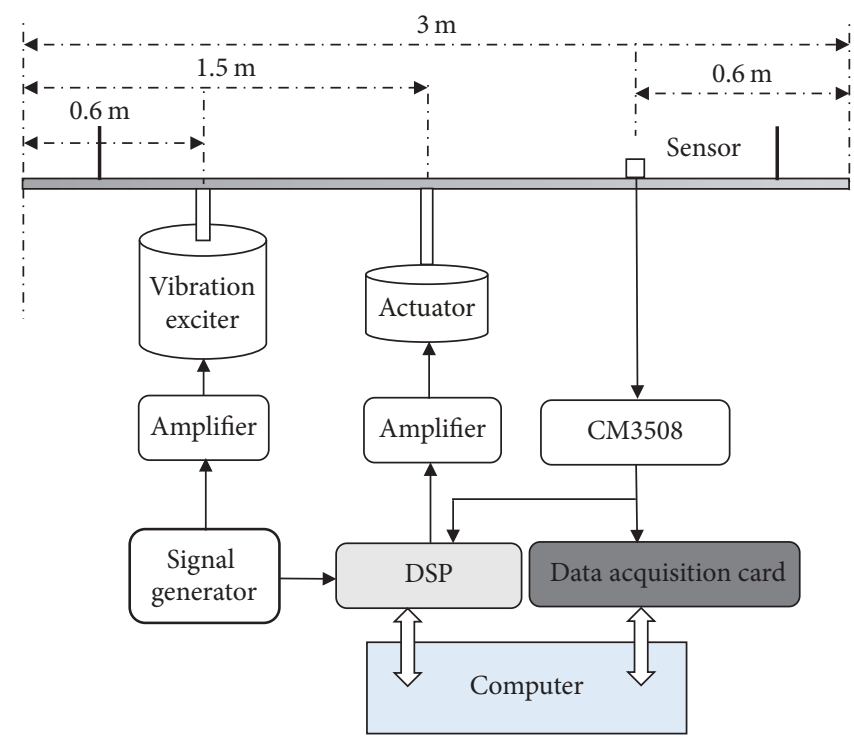

FIGURE 13: The schematic diagram of active vibration control system.
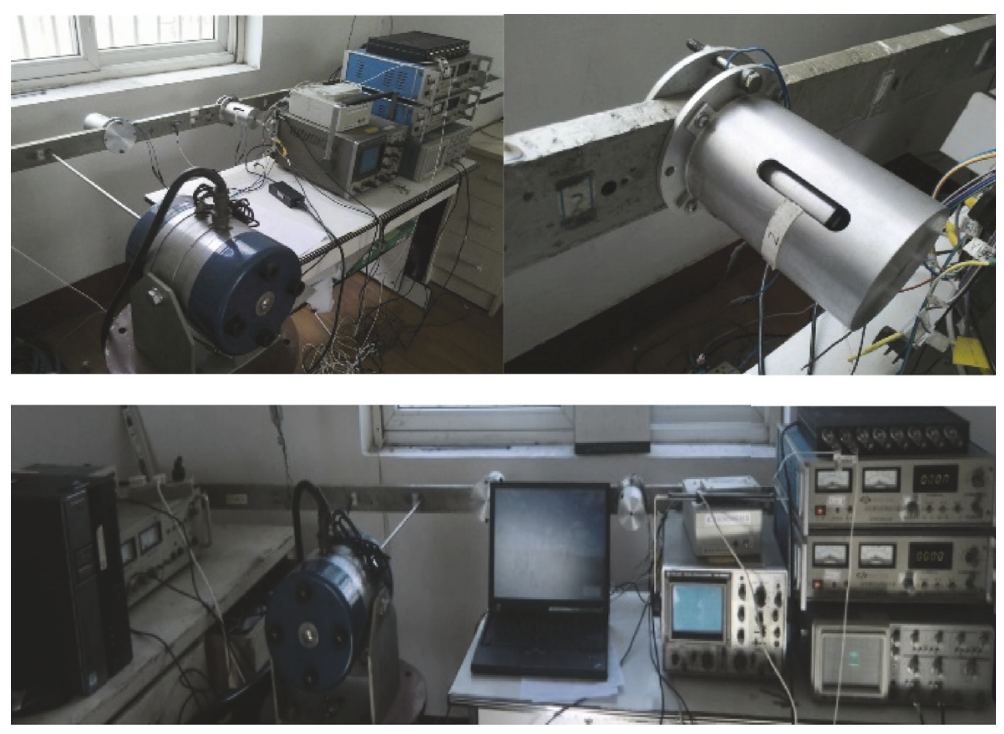

FIgURE 14: The diagram of experimental set-up.

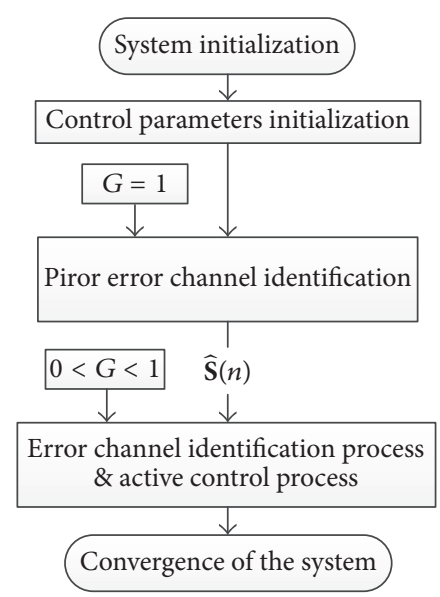

FIGURE 15: Flow chart of the program. the vibration of the controlled point is suppressed effectively and reaches a steady state after about $18 \mathrm{~s}$. The time history of the proposed method is shown in Figure 16(b). The part from 0 to $4 \mathrm{~s}$ corresponds to the uncontrolled state, and the time from $4 \mathrm{~s}$ to $7 \mathrm{~s}$ is used to identify the error channel before active control. The active controller starts working from $T=7 \mathrm{~s}$. As can be seen, the vibration is suppressed quickly, and it reaches a steady state using only about $10 \mathrm{~s}$.

Figure 17 presents the residual vibrations of Yang's method and the proposed method. The residual vibration of the proposed method is only 30\% of Yang's method.

The experimental results show the superiority of the proposed method over Yang's method in performance of vibration reduction and convergence speed. And through the experiment research the effectiveness of the proposed 


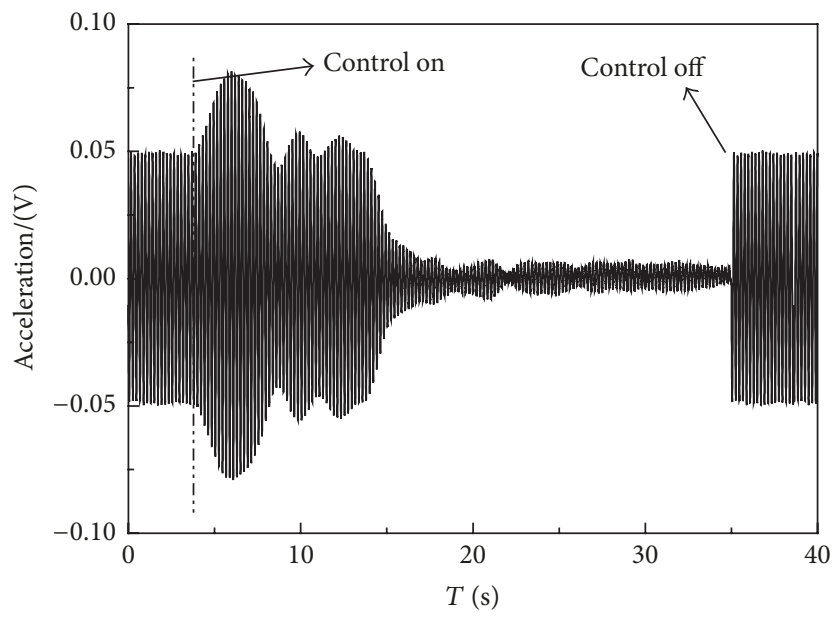

(a) Yang's method

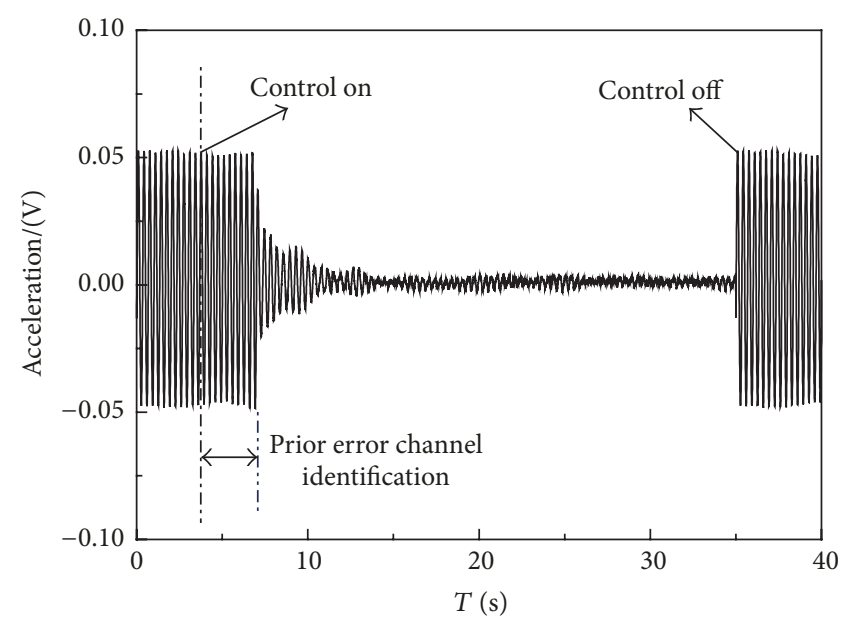

(b) Proposed method

FIGURE 16: Performance comparison between Yang's method and the proposed method.

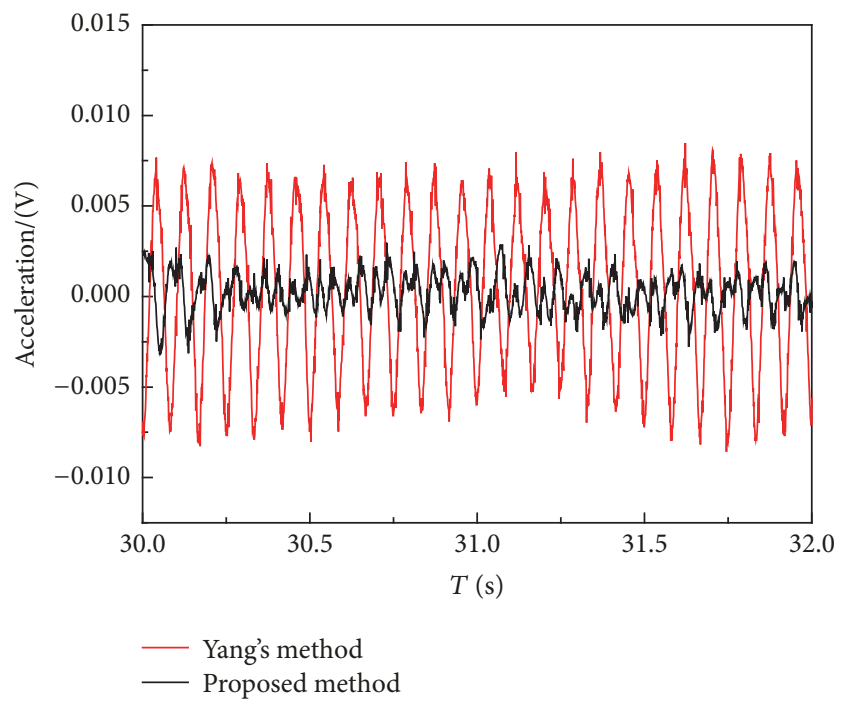

FIGURE 17: Residual vibration comparison between Yang' method and the proposed method.

method based on the prior error channel identification strategy is confirmed.

\section{Conclusions}

A new active vibration control method for helicopter based on the prior error channel identification strategy is brought out in this paper.

(i) According to the analysis of the proposed method, it can reduce the interaction between the active control process and the error channel identification process and lower computational complexity.

(ii) Simulation results show that the proposed method has lower residual vibration, faster convergence speed, and better capacity for resisting disturbance compared with the previous methods. (iii) The ACSR experiment is carried out on a freefree beam based on the embedded DSP. The results verify that the proposed method is faster than Yang's method and the residual vibration of the proposed method is only $30 \%$ of Yang's method.

\section{Conflicts of Interest}

The authors declare that they have no conflicts of interest.

\section{Acknowledgments}

The research work is supported by the National Natural Science Foundation of China (no. 51375229) and Foundation of National Key Laboratory of Rotorcraft Aeromechanics (no. 614222004021622 20003).

\section{References}

[1] C. W. Yin and G. R. Tong, "Study of the technological approaches to control helicopter vibration," Helicopter Technique, vol. 160, no. 4, pp. 26-30, 2009.

[2] G. Reichart, "Helicopter vibration control survey," Vertica, vol. 5, no. 1, pp. 1-20, 1981.

[3] S. Lei, Y. Zhang, and H. Wang, "Transmission characteristics of focul fluid elastomer vibration isolation system," Journal of Nanjing University of Aeronautics and Astronautics, vol. 47, no. 2, pp. 275-278, 2015.

[4] Y. Chen, D. G. Zimcik, V. K. Wickramasinghe, and F. Nitzsche, "Research of an active tunable vibration absorber for helicopter vibration control," Chinese Journal of Aeronautics, vol. 16, no. 4, pp. 203-211, 2003.

[5] A. M. Lin, W. T. Wang, M. Q. Li, and Y. Zhu, "Evaluation of vibration reduction efficiency for bifilar hub absorber," Helicopter Technique China, vol. 177, no. 4, pp. 1-6, 2013.

[6] C. Kessler, "Active rotor control for helicopters: motivation and survey on higher harmonic control," CEAS Aeronautical Journal, vol. 1, no. 1-4, pp. 3-22, 2011. 
[7] C. Kessler, "Active rotor control for helicopters: individual blade control and swashplateless rotor designs," CEAS Aeronautical Journal, vol. 1, no. 1-4, pp. 23-54, 2011.

[8] P. P. Friedmann and T. A. Millott, "Vibration reduction in rotorcraft using active control - A comparison of various approaches," Journal of Guidance, Control, and Dynamics, vol. 18, no. 4, pp. 664-673, 1995.

[9] P. P. Friedmann, "On-blade control of rotor vibration, noise, and performance: Just around the corner? The 33rd Alexander Nikolsky honorary lecture," Journal of the American Helicopter Society, vol. 59, no. 4, Article ID 041001, 2014.

[10] A. E. Staple and D. M. Wells, "Development and testing of an active control of structural response system for the EH101 helicopter," in Proceedings of the 16th European Rotorcraft Forum, 1990.

[11] T. Chiu and P. P. Friedmann, "An analytical model for ACSR approach to vibration reduction in a helicopter rotor-flexible fuselage system," The Aeronautical Journal, vol. 101, no. 1009, pp. 399-408, 1997.

[12] B. Vignal and T. Krysinski, "Development and qualification of active vibration control system for the Eurocopter EC225/ EC725," in Proceedings of the 61st Annual Forum Proceedings AHS International, pp. 96-106, Grapevibe, Tex, USA, June 2005.

[13] T. A. Millott, R. K. Goodman, J. K. Wong, W. A. Welsh, J. R. Correia, and C. E. Cassil, "Risk reduction flight test of a preproduction active vibration control system for the UH-60M," in Proceedings of the American Helicopter Society 59th Annual Forum, Phoenix, Ariz, USA, 2003.

[14] R. K. Goodman and T. A. Millott, "and flight testing of the active vibration control system for the Sikorsky S-92," in Proceedings of the American Helicopter Society 56th Annual Forum, Phoenix, Ariz, USA, 2000.

[15] M. Priems, S. Dreher, P. Konstanzer, and F. Hossmann, "Active cabin vibration control for light single and twin engine helicopters," in Proceedings of the American Helicopter Society 68th Annual Forum, Fort Worth, Tex, USA, 2012.

[16] F. Hoffmann, P. Konstanzer, M. Priems, and J. Chemin, "Active cabin vibration reduction for jet-smooth helicopter ride," in Proceedings of the 35th European Rotorcraft Forum 2009, ERF 2009, pp. 806-813, Hamburg, Germany, September 2009.

[17] D.-H. Kim, T.-J. Kim, S.-U. Jung, and D.-I. Kwak, "Test and simulation of an active vibration control system for helicopter applications," International Journal of Aeronautical and Space Sciences, vol. 17, no. 3, pp. 442-453, 2016.

[18] Y. Lu, Z. Q. Gu, A. M. Lin, and M. Q. Li, "Flight test of active control of structure response for helicopter," Journal of Vibration Engineering, vol. 25, no. 1, pp. 24-29, 2012.

[19] T.-J. Yang, Z.-Q. Gu, and M.-Y. Lu, "Investigation of the active control of helicopter structural vibration with online secondary path modeling," Acta Aeronautica et Astronautica Sinica, vol. 25, no. 1, pp. 36-40, 2004.

[20] L. J. Eriksson and M. C. Allie, "Use of random noise for on-line transducer modeling in an adaptive active attenuation system," The Journal of the Acoustical Society of America, vol. 85, no. 2, pp. 797-802, 1989.

[21] M. Zhang, H. Lan, and W. Ser, "A robust online secondary path modeling method with auxiliary noise power scheduling strategy and norm constraint manipulation," IEEE Transactions on Audio, Speech and Language Processing, vol. 11, no. 1, pp. 4553, 2003. 


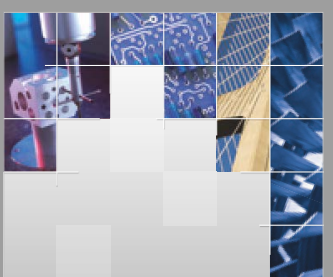

\section{Enfincering}
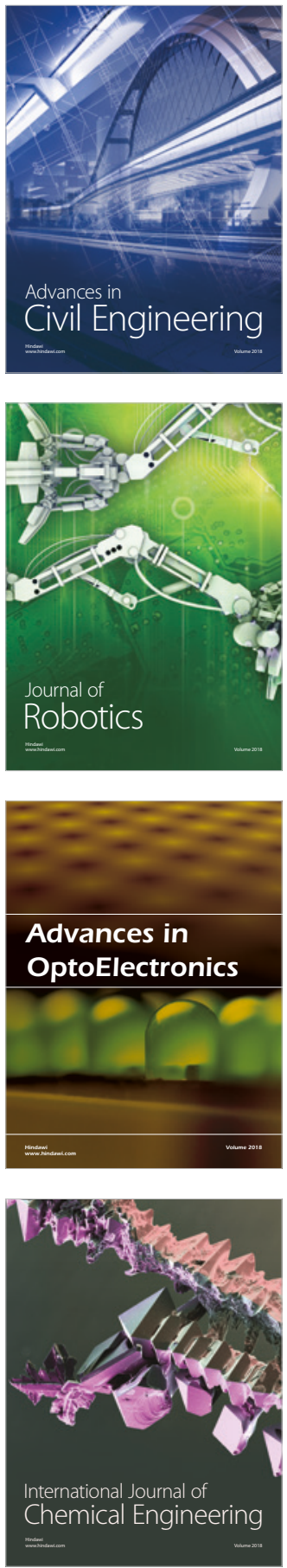

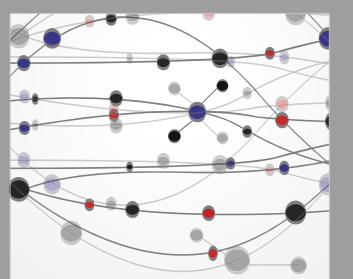

\section{Rotating \\ Machinery}

The Scientific World Journal

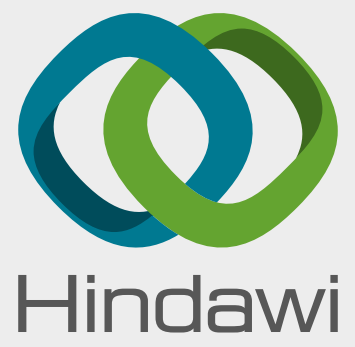

Submit your manuscripts at

www.hindawi.com
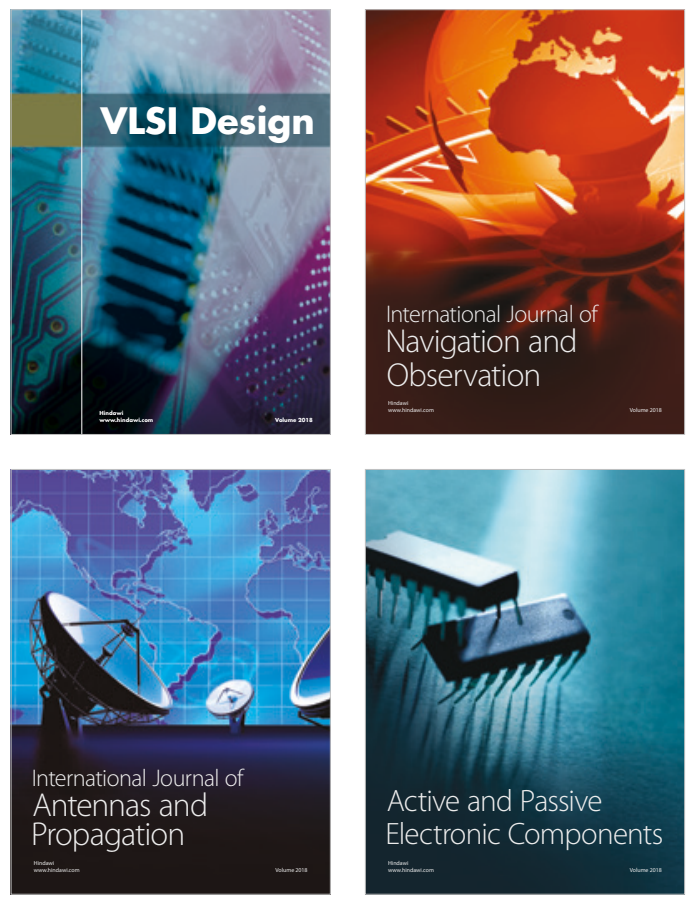
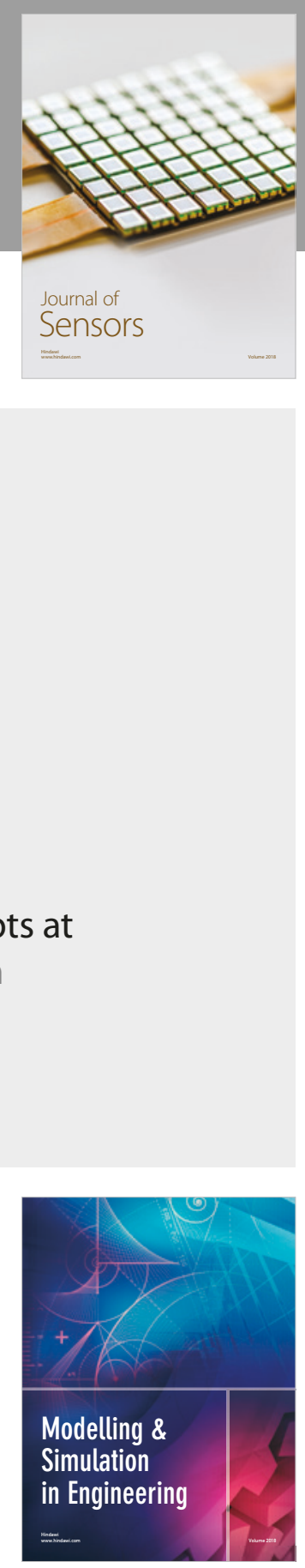

\section{Advances \\ Multimedia}
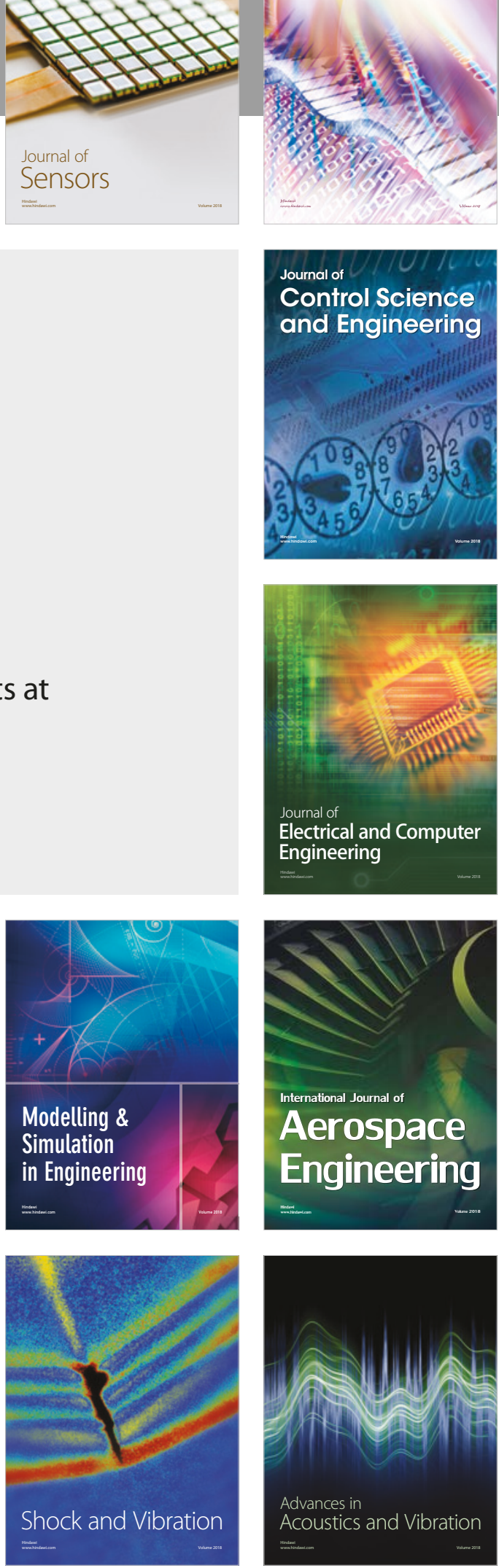